\title{
Optimization Method for PSA-based Multi-Level Regenerators
}

\author{
Mariia Sorokina $^{(*)}$, Stylianos Sygletos, and Sergei Turitsyn \\ Aston Institute of Photonic Technologies, Aston University, B4 7ET Birmingham, UK, *sorokinm@aston.ac.uk
}

\begin{abstract}
We develop an analytical methodology for optimizing phase regeneration based on phase sensitive amplification. The results demonstrate the scalability of the scheme and show the significance of simultaneous optimization of transfer function and the signal alphabet.
\end{abstract}

\section{Introduction}

Future long haul transmission systems will use complex and high spectral efficient modulation formats to meet the requirements for high capacity networking ${ }^{1}$. The use of dense constellation diagrams implies that communication systems will be more vulnerable to transmission impairments requiring frequent regeneration functionality along the link. In this respect, all-optical regenerators have a key role to play as low cost and energy efficient alternatives to opto-electronic (OEO) repeaters for improving the transparent length and the operating margins of the network system.

The capability to deal with advanced signal formats by enabling direct removal of phase distortions is imperative for such subsystems. This was made possible only recently with the development of the first multi-level optical phase quantizer ${ }^{2}$ based on phase sensitive amplifier (PSA) technology. Although single stage optimizations of the scheme have indicated operation with dense phase encoded formats ${ }^{3}$ its cascadability performance remains unknown. The true benefits of all-optical regeneration can only be realized if the nonlinear elements can co-exist along the transmission link to provide efficient suppression of the generated noise. Therefore, accurate optimization of the regenerator transfer function characteristics is required.

Here, we develop an analytical method to optimize the nonlinear response and define the operational tolerance of PSA regenerators in multi-level phase encoded transmission systems. The results demonstrate good agreement between the analytical predictions and the numerical simulations, and underline the importance of the proposed optimization method.

\section{Optimization Method}

An $M$-level all-optical phase quantizer can be practically realized by means of a phase sensitive amplifier scheme that enables coherent addition of an input signal $r e^{i \varphi}$ with its

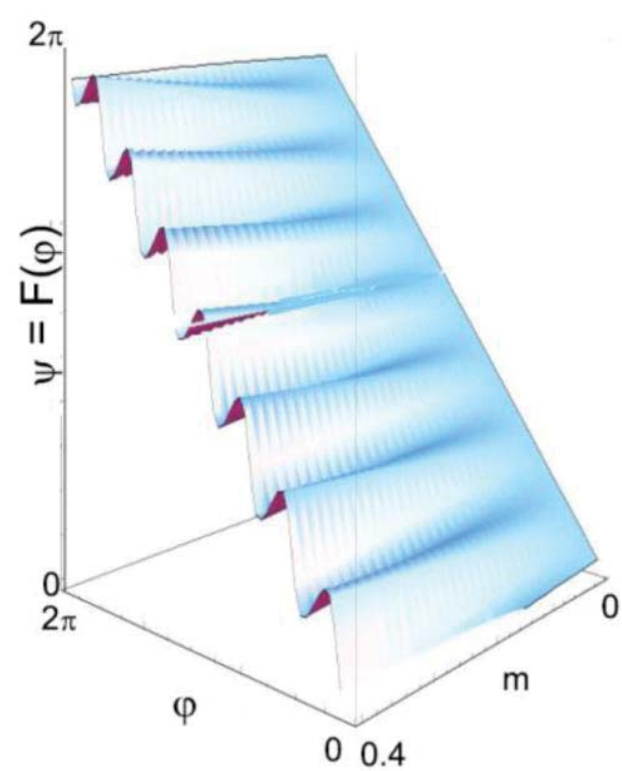

Fig. 1: Periodicity and slope dynamic of the PSA phase transfer function with varying input phase $\varphi$ and regeneration parameter $m$.

$(M-1)$ order phase conjugated harmonic, according to $R e^{i \psi}=r e^{i \varphi}\left(1+m e^{-i \varphi M}\right)$. Here, $R$ is the amplitude response of the regenerator, characterized by a sinusoidal dependence on the phase of the input signal, and $\psi=F(\varphi)$ is the staircase phase response, which can be written as:

$$
\psi=F(\varphi)=\tan ^{-1}\left(\frac{\sin [\varphi]+m \sin [\varphi(1-M)]}{\cos [\varphi]+m \cos [\varphi(1-M)]}\right)
$$

In the above equations $m$ is the optimization parameter which defines the regenerative properties of the PSA. The transfer function creates attraction regions ${ }^{4}$, which enable phase noise suppression, as depicted in Fig. 1. Clearly an optimum set of the parameter values needs to be determined for maximizing the regeneration efficiency of the overall system.

Conventional optimization methods were based on minimizing the difference between the achieved phase transfer function of the regenerator and an ideal stepped function ${ }^{3}$. 


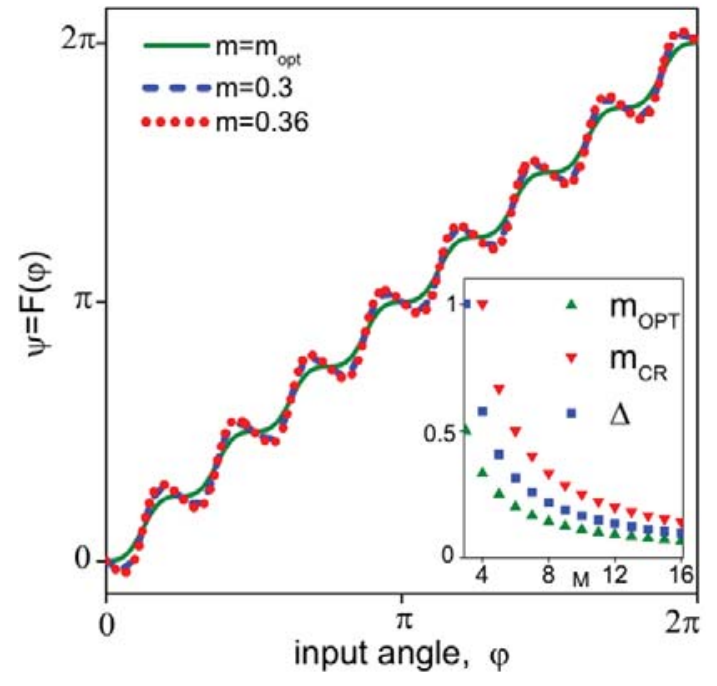

Fig. 2: Regenerative phase transfer function for various values of $m$ for 8 -PSK. The optimal value of $m$ (green solid line) shows a plateau centered at the alphabet points. Suboptimal value $m=0.3$ (blue dashed line) results in SNR improvement of $1 \mathrm{~dB}$ for single PSA and nearly $3 \mathrm{~dB}$ for 10 cascaded PSAs (for SER $=10^{-3}$ ). The non-optimal $m=0.36$ (red dotted curve) significantly degrades system performance. The inset shows the dependence of the optimal and critical values of $m$ and the plateau region $\Delta$ on the order M of PSK modulation format.

Here, we stress that a plateau is not a necessary condition for effective regeneration performance. Instead, we propose a set of simple requirements to define the signal alphabet and the operating margins of our regenerative subsystems. The scheme is general and can be applied to any regenerative sub-system ${ }^{5}$.

Firstly, it is required to adjust the signal alphabet to the nonlinear transfer function of the regenerator. The optimum position of the alphabet has to be at the centre of the corresponding attraction regions, defined by $F^{\prime \prime}\left(\varphi^{*}\right)=0$, thus the input constellation is given by $\varphi^{*}=l \pi / M, l \in Z$.

Secondly, the condition of the transformation stability (i.e. for the effective noise suppression) leads to: $\left|F^{\prime}\left(\varphi^{*}\right)\right|<1$. Calculated at the alphabet points, it gives the variation limits of the regenerative parameter $m$ :

$$
|m|<m_{c r}, \quad m_{c r}=\frac{2}{M-2}
$$

here $m>0$ if $l=2 k$ and $m<0$ if $l=2 k+1$, with $k \in Z$. The optimal value is defined by $F^{\prime}\left(\varphi^{*}\right)=0$, therefore

$$
\left|m_{\text {opt }}\right|=\frac{1}{M-1}
$$

This case results in plateau regions around the alphabet points with the width expressed by

$$
\Delta=\sqrt{\frac{2}{(M-2)(M-1)}}
$$

Fig. 2 demonstrates the optimal choice (green solid line) of $m$ that has plateau regions and suboptimal choice (blue dashed line) that does not has plateau but still gives significant signal improvement. The inset in Fig. 2 shows the optimal and critical values of the regenerative PSA parameter. One can see that for large constellations size $M$ the gap between two values is narrowed making the PSA optimization more significant. Also, the figure shows the change of the plateau width as a function of the constellation size.

The validity of the proposed analytical optimization method is confirmed by simulating numerically the performance of the transmission systems with cascaded phase regenerators.

\section{Results and Discussion}

Transmission impairments were modeled by additive Gaussian noise that was distributed uniformly along the transmission line. Without loss of generality, the regenerators were placed equidistantly. The signal to noise ratio (SNR) was defined as the ratio of the signal power to the power of the linearly added noise along the link, thus coinciding with the SNR of the equivalent linear system i.e. in the absence of regeneration.

Fig. 3 depicts the achieved SNR improvement defined at a fixed symbol error rate of (SER) $\mathrm{SER}=10^{-3}$, as a function of the parameter $m$ for different numbers of cascaded regenerators $(n=1, n=10$ and $n=20)$, and for different modulation formats (4, 8 and 16-PSK). Although 4-PSK (QPSK) format demonstrates performance gain over a wide range of values $m$, by increasing the constellation complexity the operating margins are significantly reduced. Therefore, the proposed optimization method is necessary to ensure adequate cascadability and provide higher SNR gains.

We stress that a plateau on the transfer function of the phase quantizer is not a necessary condition for demonstrating regenerative performance along the cascade. It is also possible to achieve effective noise suppression by suboptimal choices of the parameter $m$. For example, $m=0.3$ gives for8PSK an SNR improvement of $2 \mathrm{~dB}$, whereas 




Fig. 3: The dependence of the SNR improvement due to PSA application for the fixed value SER $=10^{-3}$ as a function of the PSA parameter $m$ for 4 (blue), 8 (green) and 16 (red) PSK modulation formats. The cascaded scheme of 10 (dashed) and 20 (dotted) PSA elements gives significant improvement for high order PSK formats. The optimal value of $m$ is shown by vertical lines of the corresponding color for each constellation size.

the transfer function (see blue curve on Fig.2) does not show any plateau-like behavior.

On the other hand, $m=0.36$ provides a similar transfer function (see red curve on Fig.2), but it degrades the system performance compared to the channel in the absence of PSA, as it slightly exceeds the critical value $m=0.33$, which our theory has set as a limit for regeneration.

Also, we observe the saturation effect for the cascaded scheme, i.e. increasing the number of PSA elements above a critical value gives no significant improvement to the signal transmission performance.

Fig.4 depicts the SER performance as a function of the SNR for the aforementioned cascadability scenarios and modulation formats. Results are shown also the two cases of optimal and critical values of the m-parameter. The induced degradation from a non-optimal $m$ value becomes more pronounced as the number of cascaded PSA regenerators is increased.

Finally, the significant benefit due to optimization of 10 cascaded PSA is illustrated in Fig. 5 on the example of the 8-PSK format.

\section{Conclusions}

We have proposed an analytical optimization technique to define the optimal regenerative properties and operating margins of multi-level PSA based regenerators in transmission links. Analytical conclusions have been verified by a

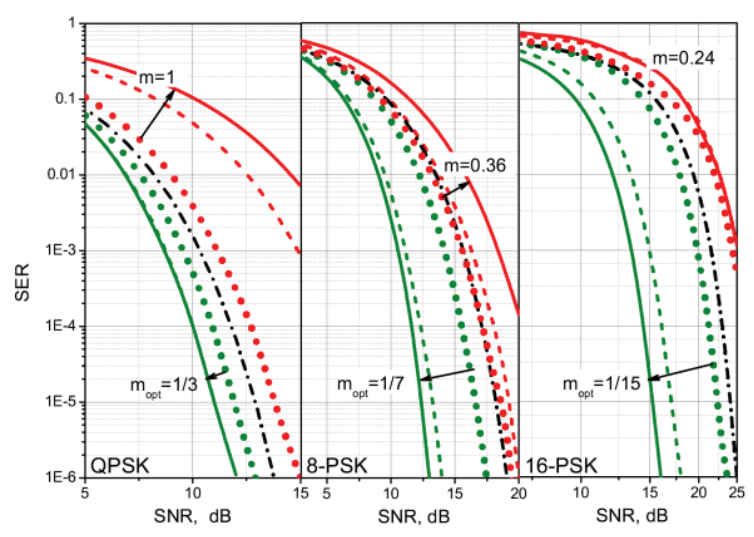

Fig. 4: SER as a function of SNR with one PSA (dotted) and cascaded scheme of 10 (dashed) and 20 (solid) PSA elements. The SER of the linear channel is shown for comparison by black dash-dotted line. The SER of the system with the optimal and critical values of the parameter $m$ are shown by green and red curves correspondingly. The arrows show the SER evolution for increasing number of PSAs.

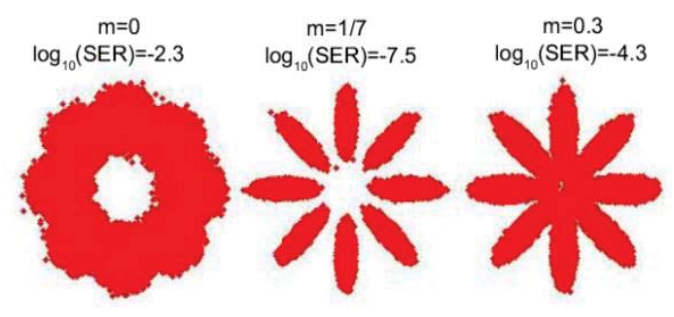

Fig. 5: Constellation diagram of 8-PSK after transmission with $\mathrm{SNR}=15 \mathrm{~dB}$ without regeneration (right panel) and improvement after 10 cascaded PSAs with the optimal $m=1 / 7 \quad$ (centre panel) and suboptimal $m=0.3$ (left panel). One can observe the dramatic gain in the system performance due to the PSA optimization for the cascaded scenario.

rigorous numerical study of various transmission scenarios and significant performance benefits have been estimated. We have shown that the optimization sensitivity is particularly significant for dense phase encoded signals. The model can be applied for high order modulation formats and different cascaded regeneration scenarios leading to dramatic improvement of the information capacity ${ }^{5}$

\section{Acknowledgements}

This work was supported by the EPSRC program grant UNLOC.

\section{References}

[1] P. Winzer et al.JLT vol.24(12) pp 4711-4728, 2006

[2] J. Kakande et al., Nature Photonics 5, 748 (2011).

[3] J. Kakande et al., Proc. OFC'12, OW1I.3 (2012).

[4] K. S. Turitsyn and S. K. Turitsyn, Opt. Lett. 37(17), 3600 (2012)

[5] M. Sorokina and S. K. Turitsyn, submitted for publication 